PAPER

Noncommutative black hole in the Finslerian spacetime

To cite this article: Sourav Roy Chowdhury et al 2021 Class. Quantum Grav. 38145019

View the article online for updates and enhancements.

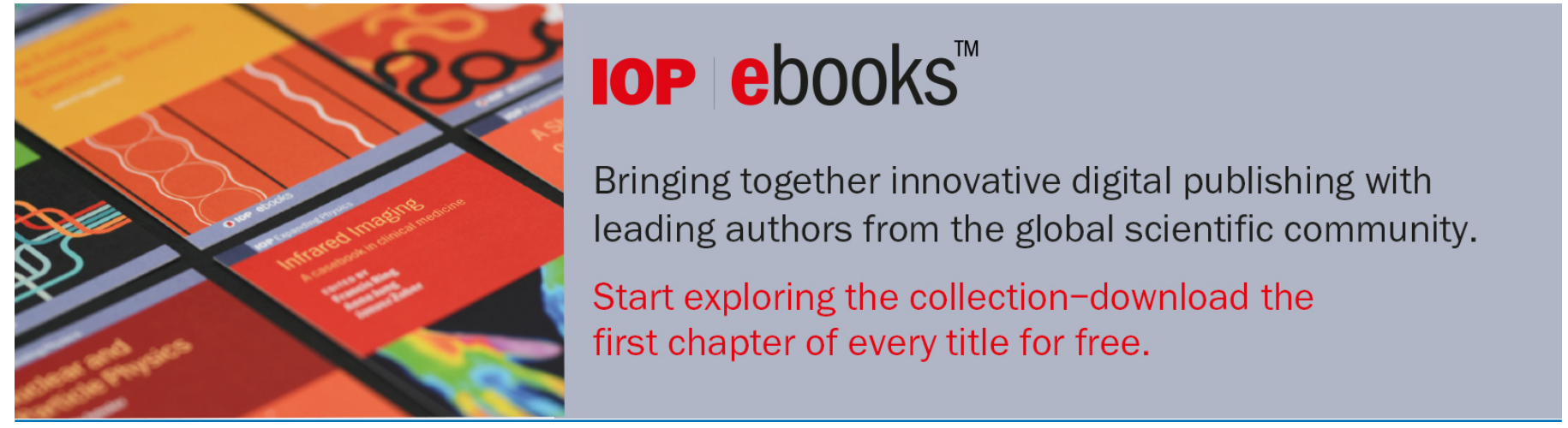

This content was downloaded from IP address 14.139 .128 .34 on 03/09/2021 at 09:11 


\title{
Noncommutative black hole in the Finslerian spacetime
}

\author{
Sourav Roy Chowdhury ${ }^{1}$, Debabrata Deb $^{2}$, \\ Farook Rahaman $^{3}\left(\mathbb{1}\right.$, Saibal Ray ${ }^{4, *}{ }^{(1)}$ and B K Guha ${ }^{1}$
}

${ }^{1}$ Department of Physics, Indian Institute of Engineering Science and Technology, B. Garden, Howrah 711103, West Bengal, India

${ }^{2}$ Department of Physics, Indian Institute of Science, Bangalore 560012, India

3 Department of Mathematics, Jadavpur University, Kolkata 700032, West Bengal, India

${ }^{4}$ Department of Physics, Government College of Engineering and Ceramic

Technology, 73 A.C.B. Lane, Kolkata 700010, West Bengal, India

E-mail: sourav.rs2016@physics.iiests.ac.in,debabratadeb@iisc.ac.in, rahaman@associates.iucaa.in, saibal@associates.iucaa.in and

bkg@physics.iiests.ac.in

Received 13 July 2020, revised 8 January 2021

Accepted for publication 5 February 2021

Published 15 June 2021

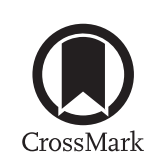

\begin{abstract}
We study the behavior of the noncommutative radiating Schwarzschild black hole in the Finslerian spacetime. The investigation shows that black hole possesses either (i) two horizons, or (ii) a single horizon, or (iii) no horizon corresponding to a minimal mass. We obtain that the minimal mass significantly changes with the Finslerian parameter, keeping minimal horizon remain unchanged. It turns out that under Finslerian spacetime, the maximum temperature before cooling down to absolute zero varies with the Finslerian parameter. We then study the stability of the black hole by analyzing the specific heat and free energy. The energy conditions, their violation limit also scrutinized. Our findings suggest a stable black hole remnant, whose mass and size are uniquely determined in terms of the Finslerian parameter $\overline{\text { Ric }}$ and noncommutative parameter $\chi$. The physical relevance of these results are discussed in a brief.
\end{abstract}

Keywords: noncommutative geometry, Finslerian spacetime, blackhole, numerical relativity

(Some figures may appear in colour only in the online journal)

*Author to whom any correspondence should be addressed. 


\section{Introduction}

In classical general relativity (GR), the notion of the black hole is as an object in spacetime, exhibiting such strong gravitational acceleration that no particles even electromagnetic radiation can escape from it. In the curved background, based on quantum field theory (QFT), Hawking has been shown that a black hole can evaporate by emitting thermal radiation analogous with the black body radiation $[1,2]$. The discovery of Hawking radiation solved the problem in black hole thermodynamics and reconciliation of the quantum mechanics and gravity. Hawking has pointed out that the virtual particles with negative energy in the vicinity of the black hole can come into the black hole through the tunneling effects. As a consequence, the energy of the system and the radius of the event horizon will decrease. Bekenstein $[3,4]$ proposed that the entropy of the black hole is proportional to the area of the horizon.

The final state of black holes evaporation issue can settle down in possible ways. As Hawking suggested, one of these may be that there is no remnant leaving behind the complete evaporation of black hole except only incoherent radiation. As a consequence, loss of quantum coherence does occur. Alternatively, stable remnants leave behind the Hawking process and in the initial configuration of the system which reflects in precise quantum states [5]. To define this, the number of distinct virtual quantum states are needed. There are two different aspects to be accounted for physical consideration. The first issue is the remnant's size and mass in the order of Planck scale, i.e. infinite density of states. If gravity in any regular way coupled with these light remnants, in the presence of the energy of the Planck Scale amount, there would be profuse production in pairs. Another point is that the Hawking process might terminate, in spite of the black holes still having macroscopic mass. That could only happen if a singularity develops on the apparent horizon of the geometry by the back reaction [6]. According to the string/black hole correspondence principle [7], at the late stage of the black hole the stringy effects cannot be neglected. Out of different conclusions of string theory, we are interested in the result in which the spacetime coordinates itself become the noncommutating operators on a $D$-brane $[8,9]$.

Usually considered spacetime continuum does not require any Lorentz invariance. The idea of Lorentz invariant discrete spacetime, i.e. spacetime quantization, long ago provided by Snyder [10]. The noncommutative behavior of the spacetime is encoded by the relation

$$
\left[\mathbf{x}^{\mu}, \mathbf{x}^{\nu}\right]=i \chi^{\mu \nu},
$$

where $\chi^{\mu \nu}$ is an anti-symmetric matrix. It determines the discretization of the cell of spacetime in the same manner as the phase space discretized by the Planck constant $\hbar$. The noncommutativity is an intrinsic parameter of spacetime and independent of the behavior of curvature $[11,12]$. The modified QFT, after implementation of the commutator relation equation (1) has been largely investigated based on two distinct approaches: (i) Wely-Winger-Moyal ${ }^{*}$-product and (ii) coordinate coherent state formalism [13]. It has been shown that the doubts raised on the Lorentz invariance and unitary $[14,15]$ in the ${ }^{*}$-product approach can figure out on considering $\chi^{\mu \nu}=\chi \operatorname{diag}\left(\epsilon_{1}, \ldots, \epsilon_{D / 2}\right)[16]$ where $\chi$ is a constant of the dimension of the order length square.

The order of the magnitude of the noncommutative parameter and its connection with the observations has also been searched by several researchers $[17,18]$. Since the noncommutativity has been well inspired by the quantum gravity and string theory, it's expected that any noncommutative corrections are in the Planck scale. Carroll et al [19] found the upper bound of the parameter $|\chi|$ is $\approx 10 \mathrm{TeV}^{-2}$ corresponding to the length scale $\sqrt{4 \times 10^{-40}} \mathrm{~m}$ for $\hbar=$ 
$c=1$. There are also different studies [20-22] that suggests the bound could be $\approx 4 \mathrm{GeV}^{-2}-$ $30, \mathrm{MeV}^{-2}$.

As an interesting possibility to describe the spacetime geometry beyond Riemannian geometry, Finsler geometry has recently been re-discovered. We study the Finslerian black holes induced as possible effects of quantum spacetime noncommutativity. Nonholonomic frames represent such Finsler structures not on tangent bundles but on (pseudo) Riemannian manifolds, compatible with standard physics theories [23]. We can extract the configuration of the black hole from two types of solutions. Nonholonomic deformations of the gravitational vacuum by noncommutative geometry are defined as the first class of solutions. The second class of solutions induces noncommutative matter fields. In this work, we emphasized constructions when black hole configurations are in the second class. There must be an intimate connection between a gravitational field quantization and a spacetime structure 'quantization' upon which the gravitational field is defined. In fact, one could argue that the gravitational field is a phenomenological manifestation of an inherent 'quantization' of the spacetime structure, even at a classical level. It could be suggested as the conjecture that the classical field associated with coordinate quantization is the gravitational field [24-26].

The notion of the spacetime loses by making the noncommutative spacetime coordinates but retains an algebraic function analog. The aim is to modify the geometry of a space as much as possible, in terms of its algebraic functions, and then generalize the corresponding differential geometry outcomes to the case of a noncommutative algebra. Drawing the differential form is equivalent to a derivative, adding the differential structure. One could introduce an algebra generated by the noncommutative terms and even a differential calculus to construct a geometry. The geometry would be a noncommutative generalization of a point $[27,28]$. The same description can introduce the gravitation field. The simplest is the formal minimul coupling procedure. One modification is to add the matter action so that the total action is extremal regarding the variation of the moving frame and the linear connection. The above prescription provides the Einstein equation [29]. In such induced noncummutative procedure, interior structure is modified according to the matter distribution. Local Lorentz symmetry breaking in noncommutative cut-off field theories and an effective description of the mechanisms are discussed in the cited literature [30, 31].

Nicolini et al [32] argued that this is not essential to introduce the effects of noncommutativity only by modifying the $r-t$ section of the metric of spherical symmetry and as a result the modified form of the Einstein field equations. Retaining the tensor part as same in the field equation, one can only modify the matter source of the system. The geometrical structure over the manifold is defined by the metric field and the strength of the field is measured by the curvature, i.e. the response of the existence of the matter distribution. It affects gravity in a subtle, indirect way [24]. In a plane, one can note that induced noncommutativity influences matter-energy and momentum distribution as well as propagation $[13,16,33]$. On the other hand, energy-momentum density determines spacetime curvature. By maintaining the standard form of the Einstein tensor in the lhs of the field equations and implementing a modified energy-momentum tensor as a source in the rhs, the impacts of noncommutativity can be taken into account [32].

Details study of the influence of noncommutativity in GR and the matter, energy and momentum distribution and propagation has been conducted by several scientists $[13,16]$. For the regular black hole, the quantum cooling process of evaporation has been studied in reference [34]. Banerjee et al [35] provide the generalized form of black hole temperature and surface gravity, and shown that the relation is valid until quantum relation is negligible. In a subsequent study [36], they provide the corrected form of area law in noncommutative geometry. Noncommutativity is sufficient to produce stable circular form, without the help of 
dark matter [37]. Between the Einstein and M $\emptyset$ ller prescription, the Einstein platform is more powerful to explain the energy for a noncommutative radiating black hole [38].

The notion of Finsler geometry based on the time measurement between two events that passes an observer is equivalent to the length which connects the events along the observer's world line. The quantification is on the tangent bundle of a homogeneous function $[39,40]$. It brings positional and directional dependent behavior, along with the intrinsic local anisotropy [41].

Riemann introduced a metric structure, based on arc element in general space as

$$
x \mathrm{~d} s=\mathcal{F}\left(x_{1},:: x_{n} ; y_{1},:: y_{n}\right)
$$

where $\mathcal{F}(x ; y)$ is a positive homogeneous function of degree one in $y$. Here, $x$ and $y$ representing the position vector and tangent vector respectively. However, without any the quadratic restriction, the easiest description of Riemannian geometry is in reference [39] as follows

$$
\mathcal{F}^{2}=g_{\mu \nu} y^{\mu} y^{\nu}
$$

The current Finsler space $\overline{\mathcal{F}^{2}}$ is quadratic in $\left(y^{\theta}\right.$ and $\left.y^{\phi}\right)$ which can be resolved from a Riemannian manifold $\left(\mathbb{M}, g_{\mu \nu}(x)\right)$. It is actually a semi-definite Finsler space and for that one can use the covariant derivative of the Riemannian space. The Bianchi identities, in this case, are similar to those of the Riemannian space and the current Finsler space reduces to the Riemannian space and as a result, the gravitational field equations can be found.

In the semi-Riemannian geometry, in local coordinates the length of a curve $\gamma$ on a manifold is given by

$$
L_{\mathrm{SR}}[\gamma]=\int_{t_{0}}^{t_{1}} \mathrm{~d} t \sqrt{g_{\mu \nu}(x(t)) \dot{x}^{\mu} \dot{x}^{\nu}}
$$

In Finslerian geometry, the metric geometry (including the Lorentz metric) can be extrapolated by defining a length for the curve. Instead of the metric, a general length measurement for curves on $\mathbb{M}$ derives the geometry. The geometry is engaged to matter dynamics and on assumption that the metric tensor is independent of dynamics, the system reduces to Riemannian.

As a possible alternative spacetime model, Finslerian modification is already considering in cosmology, GR. The notion of Finsler geometry is developed from quantum gravity ideas [42]. Later on, in special relativity, it is testified by Gibbons [43]. Timelike and null geodesics as well as the geodesic motion of particles on the cosmologically symmetric spacetime studied by Hohmann [44]. Finsler geometry of Ransder space and Conformal geometry in classical spacetime share several similarities, available in the references [45-47].

In reference [23, 48], Vacaru constructed a new class of exact solutions with generic offdiagonal metrics depending on a noncommutative parameter and shown that the Finsler models defined by nonholonomic frames are not on tangent bundles but on (pseudo) Riemannian manifolds being compatible with standard theories of physics. Effective energy-momentum tensors can induce by noncommutative deformations of Schwarzschild metrics into locally anisotropic stationary in spherical/rotoid symmetry. Lagrangian geometry and Finsler-Affine gravity effects elaborately studied elaborately in the monograph [49].

In this article, we report on the characteristics and behavior of the black hole inspired by noncommutative geometry in the framework of semi-Riemannian spacetime with constant flag curvature. 


\section{The interiour structure}

Let us consider that $\mathcal{F}$ is a Finsler metric on a manifold $\mathbb{M}$ which is defined as $\mathcal{F}=\mathcal{F}(x, y)$ and is a function of $\left(x^{\mu}, y^{\mu}\right)$ in $\in T \mathbb{M}$, in a standard coordinate system.

The geodesic of the Finsler metric $(\mathcal{F})$ is characterized by

$$
\frac{\mathrm{d}^{2} x^{\mu}}{\mathrm{d} \tau^{2}}+2 G^{\mu}(x, y)=0
$$

where the geodesic spray is

$$
G^{\mu}=\frac{1}{4} g^{\mu \nu}\left(\frac{\partial^{2} \mathcal{F}^{2}}{\partial x^{\lambda} \partial y^{\nu}} y^{\lambda}-\frac{\partial \mathcal{F}^{2}}{\partial x^{\nu}}\right) .
$$

The metric structure coefficient can be written in the form

$$
g_{\mu \nu}=\frac{\partial}{\partial y^{\mu}} \frac{\partial}{\partial y^{\nu}}\left(\frac{1}{2} \mathcal{F}^{2}\right) .
$$

Ricci scalar of the Finsler geometry [39] can be written as

$$
\text { Ric }=R^{\mu}{ }_{\mu}=\frac{1}{\mathcal{F}^{2}}\left[2 \frac{\partial G^{\mu}}{\partial x^{\mu}}-y^{\lambda} \frac{\partial^{2} G^{\mu}}{\partial x^{\lambda} \partial y^{\mu}}+2 G^{\lambda} \frac{\partial^{2} G^{\mu}}{\partial y^{\lambda} \partial y^{\mu}}-\frac{\partial G^{\mu}}{\partial y^{\lambda}} \frac{\partial G^{\lambda}}{\partial y^{\mu}}\right],
$$

where $R^{\mu}{ }_{\mu}$ is insensitive to connections which only depends on Finsler structure.

Ricci tensor of Finsler geometry is introduced by Akbar-Zadeh [50] as follows

$$
\operatorname{Ric}_{\mu \nu}=\frac{\partial^{2}}{\partial y^{\mu} \partial y^{\nu}}\left(\frac{1}{2} \mathcal{F}^{2} \operatorname{Ric}\right) .
$$

We consider the line element to describe the static, spherically symmetric interior spacetime is in the form

$$
\mathcal{F}^{2}=-\mathrm{e}^{\nu(r)} y^{t} y^{t}+\mathrm{e}^{\lambda(r)} y^{r} y^{r}+r^{2} \overline{\mathcal{F}}^{2}\left(\theta, \phi, y^{\theta}, y^{\phi}\right) .
$$

Here $\mathcal{F}=\mathcal{F}(x, y)$ is the Finsler metric on a manifold $\mathbb{M}$, is a function of $\left(x^{\mu}, y^{\mu}\right)$ in a standard coordinate system.

In the following ansatz we consider the angular coordinate as $\overline{\mathcal{F}}^{2}\left(\theta, \phi, y^{\theta}, y^{\phi}\right) . \overline{\mathcal{F}}$ is not dependent on $y^{t}$ and $y^{r}$, and therefore $\overline{\text { Ric }}$ is independent of $r$ derived from $\overline{\mathcal{F}}^{2}$ (in the same manner as defined in equation (5) for $\overline{\mathcal{F}}$ ). As a consequence two-dimensional Finsler space $\overline{\mathcal{F}}$ is a constant flag curvature space. In Riemannian geometry, the flag curvature is a generalization of sectional curvature. This is the simplest approach of the semi-Finslerain system.

Therefore, with the help of equation (4), the Finsler metric potential can be written as

$$
g_{\mu \nu}=\operatorname{diag}\left(-\mathrm{e}^{\lambda(r)}, \mathrm{e}^{\nu(r)}, r^{2} \bar{g}_{i j}\right),
$$

where metric potential $\bar{g}_{i j}$ derived from $\overline{\mathcal{F}}^{2}$.

Respective geodesics sprays are as follows

$$
\begin{aligned}
G^{t} & =\frac{1}{2} \lambda^{\prime} y^{t} y^{r}, \\
G^{r} & =\frac{1}{4}\left(\nu^{\prime} y^{r} y^{r}+\lambda^{\prime} \mathrm{e}^{\lambda-\nu} y^{t} y^{t}-2 r \mathrm{e}^{-\nu} \overline{\mathcal{F}}^{2}\right),
\end{aligned}
$$




$$
\begin{aligned}
G^{\theta} & =\frac{1}{r} y^{\theta} y^{r}+\bar{G}^{\theta}, \\
G^{\phi} & =\frac{1}{r} y^{\theta} y^{r}+\bar{G}^{\phi},
\end{aligned}
$$

where $\bar{G}^{\mu}$ corresponds the geodesic spray of $\overline{\mathcal{F}}^{2}$.

On considering the values of $G^{\mu}$ from equations (9)-(12) and substituting in equation (5), we obtain

$$
\begin{aligned}
\mathcal{F}^{2} \mathrm{Ric}= & {\left[\frac{\lambda^{\prime \prime}}{2}+\frac{\lambda^{\prime 2}}{4}-\frac{\lambda^{\prime} \nu^{\prime}}{4}+\frac{\lambda^{\prime}}{r}\right] \mathrm{e}^{(\lambda-\nu)} y^{t} y^{t}+\left[-\frac{\lambda^{\prime \prime}}{2}-\frac{\lambda^{\prime 2}}{4}+\frac{\lambda^{\prime} \nu^{\prime}}{4}+\frac{\nu^{\prime}}{r}\right] y^{r} y^{r} } \\
& +\left(\overline{\operatorname{Ric}}-\mathrm{e}^{-\nu}+\frac{r \nu^{\prime} \mathrm{e}^{-\nu}}{2}-\frac{r \lambda^{\prime} \mathrm{e}^{-\nu}}{2}\right) \overline{\mathcal{F}}^{2}
\end{aligned}
$$

On the other hand, the scalar curvature is defined as $S=g^{\mu \nu} \operatorname{Ric}_{\mu \nu}$.

Therefore, the Einstein tensor in Finsler spacetime takes the modified form

$$
G_{\mu \nu}=\operatorname{Ric}_{\mu \nu}-\frac{1}{2} g_{\mu \nu} S
$$

The explicit form we have

$$
S=\mathrm{e}^{-\nu}\left[\lambda^{\prime \prime}+\frac{\lambda^{\prime 2}}{2}-\frac{\lambda^{\prime} \nu^{\prime}}{2}+\frac{\lambda^{\prime}-\nu^{\prime}}{r}\right]-\frac{2}{r^{2}}\left[\overline{\operatorname{Ric}}-\mathrm{e}^{-\nu}+\frac{r \mathrm{e}^{-\nu}}{2}\left(\nu^{\prime}-\lambda^{\prime}\right)\right]
$$

The energy-momentum tensor for the fluid distribution can be written in the form

$$
T_{\nu}^{\mu}=-\left(\rho_{\chi}+p_{\mathrm{r}}\right) u^{\mu} u_{\nu}+p_{\mathrm{t}} \delta_{\nu}^{\mu}+\left(p_{\mathrm{t}}-p_{\mathrm{r}}\right) v^{\mu} v_{\nu}
$$

where $\rho_{\chi}, p_{\mathrm{r}}$ and $p_{\mathrm{t}}$ represent the energy density, radial and tangential pressures, respectively for the fluid system whereas $u_{\nu}$ and $v_{\nu}$ represent the four-velocity and radial four-vector, respectively.

Following the notion of GR, the field equations in the Finsler space-time geometry can be provided as

$$
G^{\mu}{ }_{\nu}=8 \pi T^{\mu}{ }_{\nu}
$$

For a static and spherically symmetric metric, the energy-momentum tensor is given by $T_{\nu}^{\mu}=\operatorname{diag}\left(-\rho_{\chi}, p_{\mathrm{r}}, p_{\mathrm{t}}, p_{\mathrm{t}}\right)$. The corresponding Einstein field equations can be written as

$$
\begin{aligned}
& \frac{\lambda^{\prime} \mathrm{e}^{-\lambda}}{r}-\frac{\mathrm{e}^{-\lambda}}{r^{2}}+\frac{\overline{\mathrm{Ric}}}{r^{2}}=8 \pi \rho_{\chi}, \\
& \frac{\nu^{\prime} \mathrm{e}^{-\lambda}}{r}+\frac{\mathrm{e}^{-\lambda}}{r^{2}}-\frac{\overline{\mathrm{Ric}}}{r^{2}}=8 \pi p_{\mathrm{r}}, \\
& \mathrm{e}^{-\lambda}\left[\frac{\nu^{\prime \prime}}{2}+\frac{\nu^{\prime 2}}{4}-\frac{\nu^{\prime} \lambda^{\prime}}{4}+\frac{\nu^{\prime}-\lambda^{\prime}}{2 r}\right]=8 \pi p_{\mathrm{t}} .
\end{aligned}
$$

In flat spacetime, point-like structures are eliminated by noncommutativity and replace with smeared objects [13]. Mathematically, the effects of smearing can be implemented by 


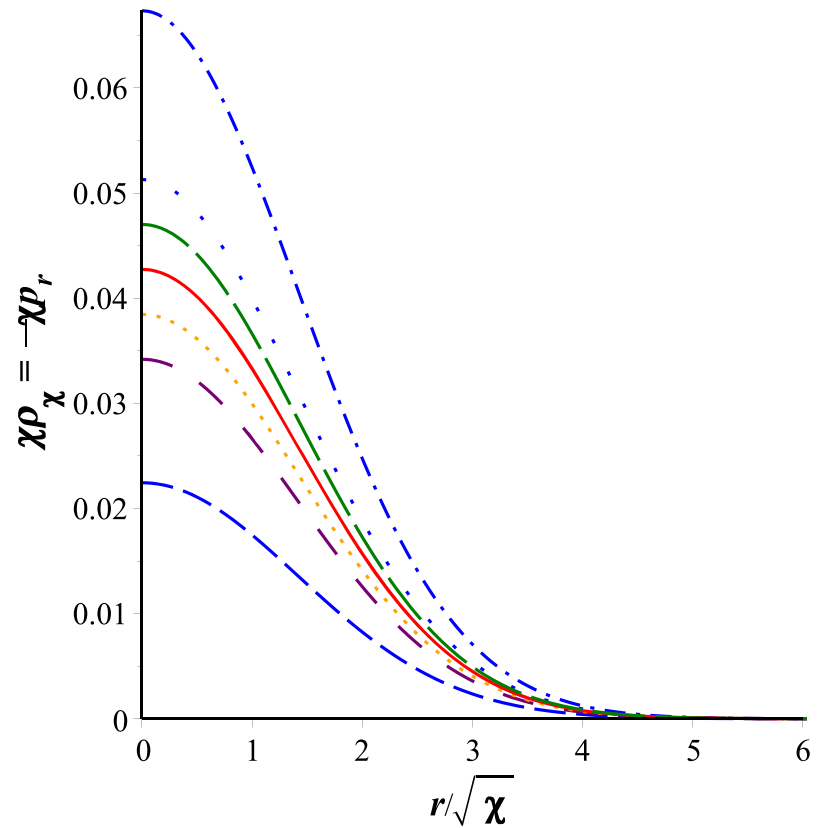

Figure 1. Variation of $\chi_{\rho}=-\chi_{p_{\mathrm{r}}} \mathrm{vs} r \sqrt{\chi}$. Line $\operatorname{dot}$ for $M=\sqrt{\chi}$, Space line for $M=3 \sqrt{\chi}$, Long dot stands: $M=2.285 \sqrt{\chi}$, Long dash stands: $M=2.094 \sqrt{\chi}$, Solid line stands: $M=1.905 \sqrt{\chi}$, Dot stands: $M=1.713 \sqrt{\chi}$, Space dash stands: $M=1.522 \sqrt{\chi}$; Blue stands: $\overline{\operatorname{Ric}}=1.2$, Green stands: $\overline{\operatorname{Ric}}=1.1$, Red stands: $\overline{\text { Ric }}=1.0$, Orange stands: $\overline{\mathrm{Ric}}=0.9$, Burgundy stands: $\overline{\mathrm{Ric}}=0.8$. Hereafter, color and line style maintain the corresponding $\overline{\mathrm{Ric}}$ and mass.

the 'substitution rule': as a position Dirac-delta function replaced by Gaussian distribution of minimal width $\sqrt{\chi}$. In connection to that, following Nicolini et al [32]. We choose the mass density for the static, smeared and particle-like gravitational source anisotropic fluid distribution as

$$
\rho_{\chi}=\frac{M}{(4 \pi \chi)^{3 / 2}} \exp \left(-r^{2} / 4 \chi\right)=-p_{\mathrm{r}}
$$

where the total mass $(M)$ of the source, instead of being perfectly localized at the point is diffused over $\sqrt{\chi}$ sized linear region. Minimal deviation from standard vacuum Schwarzschild geometry can be expected at a large distance, as well as in the range $r \simeq \sqrt{\chi}$.

Consider the variation of matter density in figure 1 . In the vicinity of origin $(r \ll \sqrt{\chi})$, the variation of matter density is almost flat, i.e. $\frac{\mathrm{d} \rho_{\chi}}{\mathrm{d} r} \simeq 0$. Again far away from the origin $(r \gg 4 \sqrt{\chi})$, the variation is also flat; $\rho_{\chi}(0) \gg \rho_{\chi}\left(r^{\prime}\right)$. Central density $\left(\rho_{\chi}(0)\right)$ is higher for higher total mass.

The conservation equation for the Finsler system is derived by Li and Chang [51]. The explicit form of the equilibrium equation for the constant flag curvature system is derived by Chowdhury et al [52]. The form remain conserved in noncommutative system [29]. We have the conservation equation as follows:

$$
\left(\rho_{\chi}+p_{\mathrm{r}}\right) \frac{\nu^{\prime}}{2}+p_{\mathrm{r}}^{\prime}-\frac{2}{r}\left(p_{\mathrm{t}}-p_{\mathrm{r}}\right)=0 .
$$




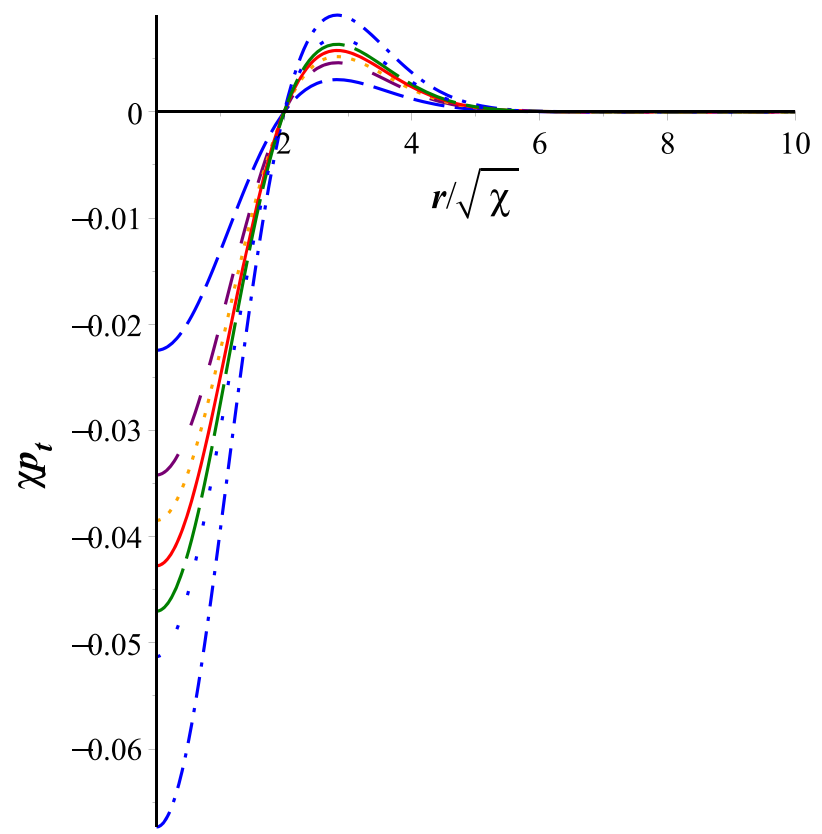

Figure 2. Variation of $\chi_{p_{\mathrm{t}}}$ vs $r \sqrt{\chi}$ for different values of $\overline{\text { Ric. }}$

For physically validity, the inward pull must balanced by non-vanishing radial pressure. This is the effect by the noncommutative spacetime on the matter at a distance scale of order $\sqrt{\chi}$. From relation equations (21) and (22), we obtain

$$
p_{\mathrm{t}}=\frac{M}{(4 \pi \chi)^{3 / 2}}\left(\frac{r^{2}}{4 \chi}-1\right) \mathrm{e}^{-r^{2} / 4 \chi}
$$

Variation of the above parameter is shown in figure 2.

We obtain the following metric coefficients for the matter distribution provided in equation (21) for the line element in equation (7)

$$
\begin{aligned}
& \mathrm{e}^{\nu}=1-\frac{4 M}{r \sqrt{\pi} \overline{\operatorname{Ric}}} \gamma\left(\frac{3}{2}, \frac{r^{2}}{4 \chi}\right), \\
& \mathrm{e}^{-\lambda}=\overline{\operatorname{Ric}}-\frac{4 M}{r \sqrt{\pi}} \gamma\left(\frac{3}{2}, \frac{r^{2}}{4 \chi}\right),
\end{aligned}
$$

where $\gamma\left(\frac{3}{2}, \frac{r^{2}}{4 \chi}\right)$ is the lower incomplete gamma function [provided in equation (A.2)].

As $\chi \rightarrow 0$, the commutative Schwarzschild metric is obtained from the noncommutative metric. Exterior Schwarzschild metric is provided in reference [51]. The variation of metric potentials $\mathrm{e}^{\nu}$ and $\mathrm{e}^{-\lambda}$ are shown in figure 3, left panel and right panel respectively. From plots it is clear that there are two horizons, (i) the inner horizon (Cauchy horizon) $r_{\mathrm{c}}$ and (ii) the outer horizon (event horizon) $r_{\mathrm{H}}$. There exists a minimal mass $M_{\mathrm{o}}$ below which no horizon, i.e. no black hole can form (space dot line). Again two distinct horizon formed for $M \gg M_{\mathrm{o}}$ (dash line). For $M=M_{\mathrm{o}}$, the inner and outer both horizons met, and one degenerate horizon (minimal horizon) found at that point $\left(r_{\mathrm{c}} \leqslant r_{\mathrm{O}} \leqslant r_{\mathrm{H}}\right)$. It is found from the plots that for the 

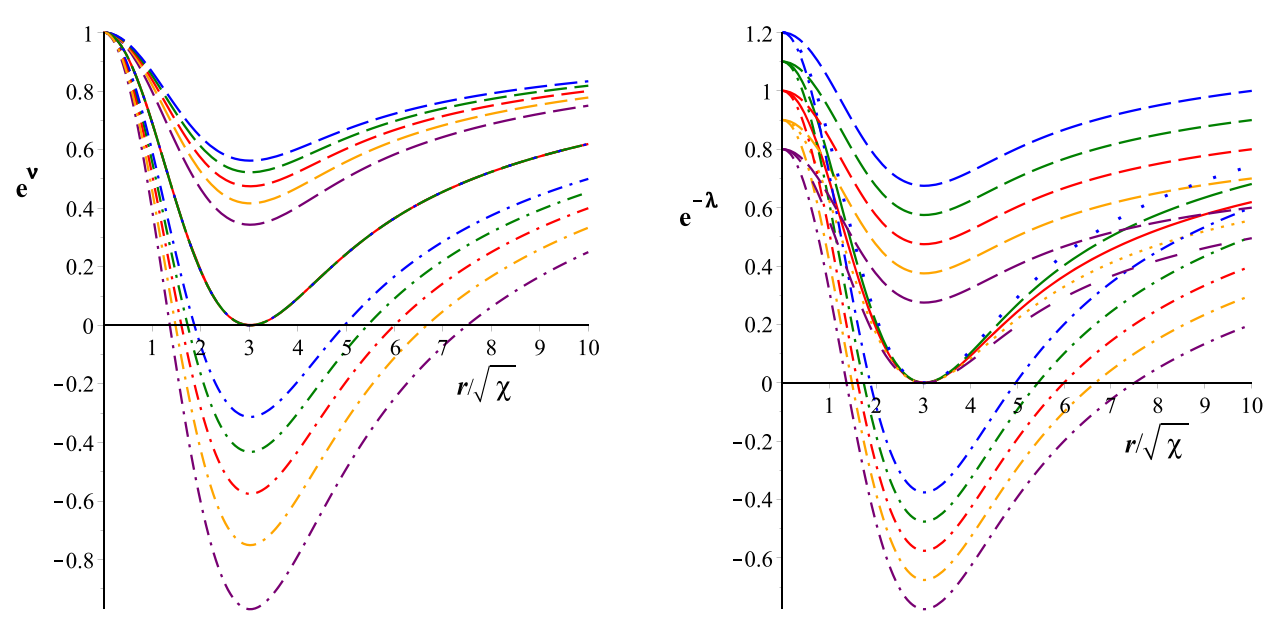

Figure 3. Variation of $\mathrm{e}^{\nu}$ and $\mathrm{e}^{-\lambda}$ vs $r \sqrt{\chi}$ for different values of $\overline{\text { Ric }}$ in the left and right panel respectively.

varying $\overline{\operatorname{Ric}}$, the degenerate horizon remain the same. Though, the minimal mass changes with $\overline{R i c}$. Higher $\overline{\text { Ric }}$ needed higher minimal mass to form a single degenerate event horizon.

The mass distribution of the system can directly obtained with the help of equation (21), as follows:

$$
m(r)=\frac{2 M}{\sqrt{\pi}} \gamma\left(\frac{3}{2}, \frac{r^{2}}{4 \chi}\right)
$$

\section{The characteristics of the black hole}

In this Section, we study few characteristics and stability of the black hole.

The event horizon of the black hole can found from equation (24) or (25), by considering $\mathrm{e}^{\nu}=0=\mathrm{e}^{-\lambda}$ in the following form,

$$
r_{\mathrm{H}}=\frac{4 M}{\sqrt{\pi} \overline{\mathrm{Ric}}} \gamma\left(\frac{3}{2}, \frac{r^{2}}{4 \chi}\right) .
$$

Solution of equation (26) cannot be obtained in closed form. Numerically, the value of $r_{\mathrm{H}}$ found from the intersection of $\mathrm{e}^{\nu}$ or $\mathrm{e}^{-\lambda}$ with the $r / \sqrt{\chi}$ for minimal mass, as shown in figure 3, left and right panel respectively. For the range $r_{\mathrm{H}}^{2} / 4 \chi \gg 1$, the lower incomplete gamma function can be expanded in the form provided in equation (A.4) by iteration. Keeping up to first order term, we find

$$
r_{\mathrm{H}} \simeq \frac{2 M}{\overline{\mathrm{Ric}}}\left(1-\frac{2 M}{\sqrt{\pi \chi}} \mathrm{e}^{-M^{2} / \chi}\right)
$$

There are different approaches also available in introducing of noncommutativity in curved spacetime metric in references [53-55]. 


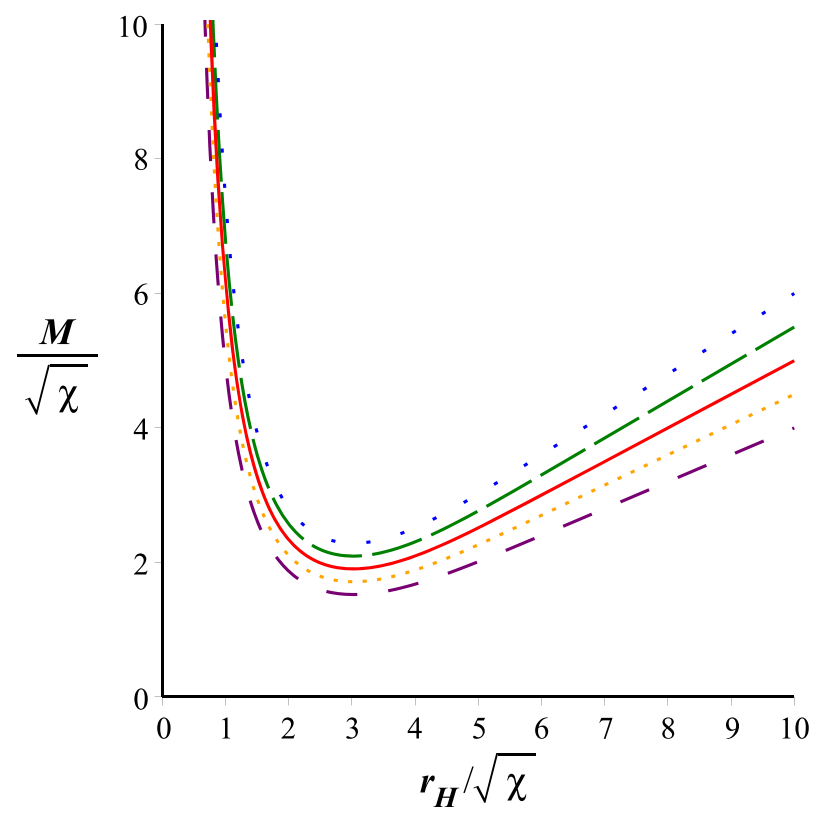

Figure 4. Variation of $M / \sqrt{\chi}$ vs $r_{\mathrm{H}} / \sqrt{\chi}$ for different values of $\overline{\text { Ric. }}$

We can readily rewrite the mass as a function of $r_{\mathrm{H}}$ :

$$
M=\frac{r_{\mathrm{H}} \overline{\operatorname{Ric}} \sqrt{\pi}}{4 \gamma\left(\frac{3}{2}, \frac{r^{2}}{4 \chi}\right)} .
$$

The variation of the total mass is shown in figure 4 . The minimal mass $M_{\mathrm{o}}$ for different values of $\overline{\mathrm{Ric}}$ is explicitly shown in figure 4 , which increases with the $\overline{\mathrm{Ric}}$. This minimal mass also confirms the degenerate horizon obtained in figure 3, left and right panel respectively.

For the static noncommutative black holes, the Hawking temperature is defined as,

$$
\begin{aligned}
T_{\mathrm{H}} & =\left.\frac{1}{4 \pi} \frac{\mathrm{de}^{\nu}}{\mathrm{d} r} \sqrt{\mathrm{e}^{\nu} \mathrm{e}^{\lambda}}\right|_{r=r_{\mathrm{H}}} \\
& =\frac{1}{4 \pi r_{\mathrm{H}} \sqrt{\operatorname{Ric}}}\left(1-\frac{r_{\mathrm{H}}^{3} \mathrm{e}^{-r_{\mathrm{H}}^{2} / 4 \chi}}{4 \chi^{3 / 2} \gamma\left(\frac{3}{2}, \frac{r^{2}}{4 \chi}\right)}\right) .
\end{aligned}
$$

For large regime $\left(r^{2} / 4 \chi \gg 1\right)$, one can easily recover the standard form of Hawking temperature

$$
T_{\mathrm{H}}=\frac{1}{4 \pi r_{\mathrm{H}} \sqrt{\mathrm{Ric}}}
$$

From figure 5 (left panel), it is clear that in the commutative consideration, Hawking temperature diverges, which impose a limit on the standard description of Hawking radiation. For the region $r<r_{\mathrm{o}}$, there is no black hole, therefore it is impossible to define temperature in that region. At the initial stage, $T_{\mathrm{H}}$ reaches maximum from $r_{\mathrm{H}}=r_{\mathrm{o}}$ instead of exploding with $r_{\mathrm{H}}$ up to the $r_{\mathrm{H}} \approx 4.8 \sqrt{\chi}$, and for $r_{\mathrm{H}} \gg r_{\mathrm{o}}$, the temperature is the same as of standard Hawking 

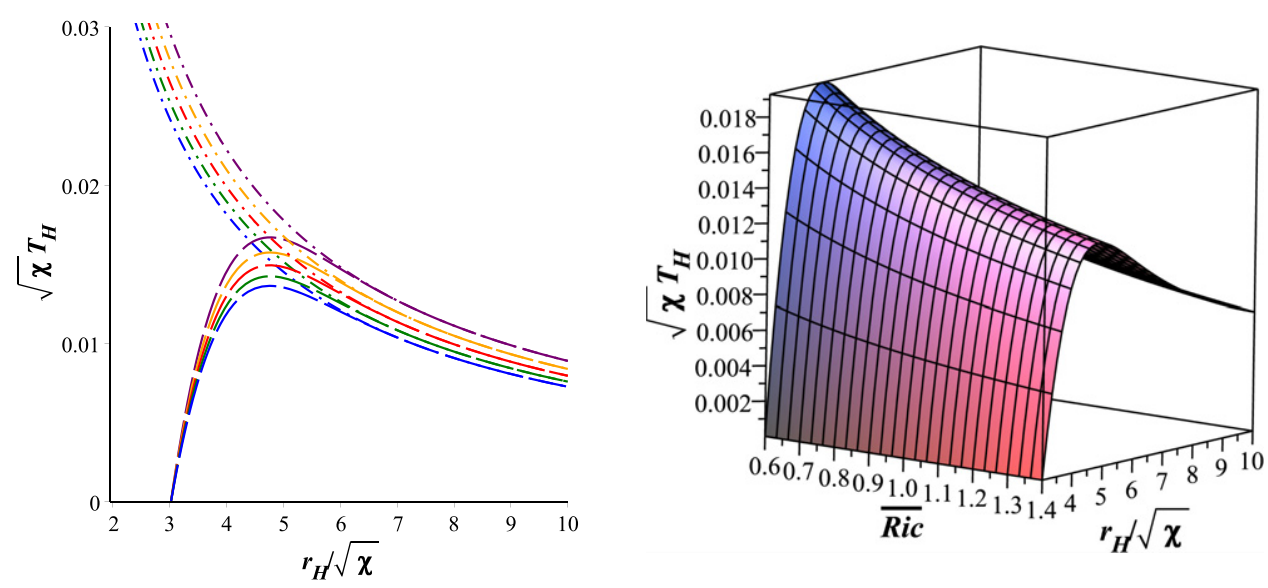

Figure 5. Variation of $T_{\mathrm{H}}$ vs $r_{\mathrm{H}}$ for different values of $\overline{\text { Ric }}$ (left panel). Here, dashdot and longdash linestyle represent commutative and noncommutative spacetime, respectively. The variation of $T_{\mathrm{H}}$ with $\overline{\mathrm{Ric}}$ for the parametric values of $r_{\mathrm{H}} / \sqrt{\chi}$ shown in the right panel.

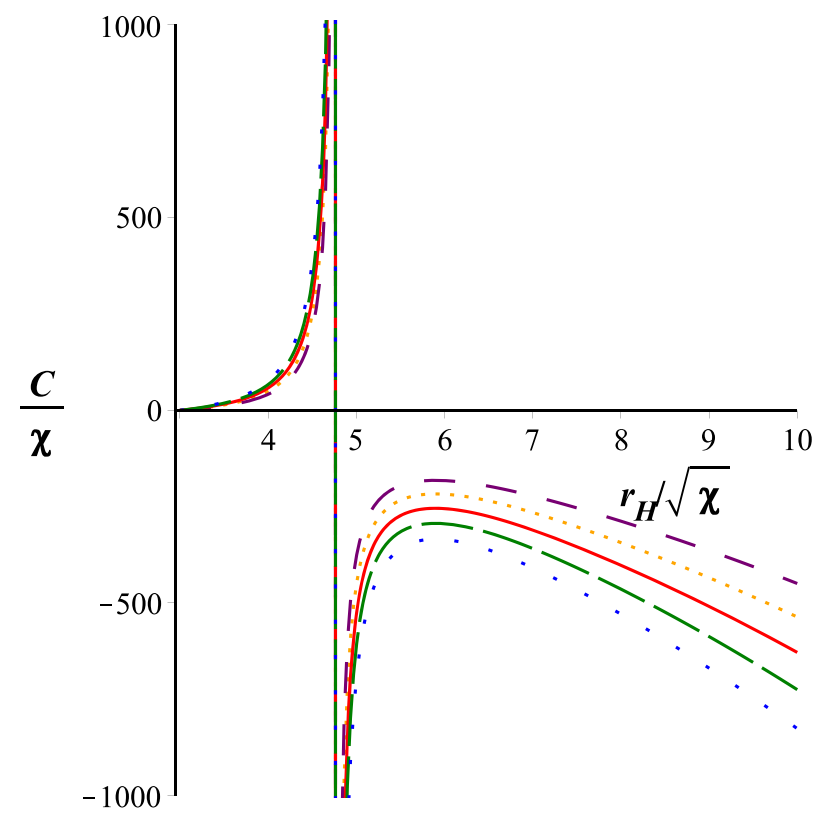

Figure 6. Plot of $C / \chi$ vs $r_{\mathrm{H}} / \sqrt{\chi}$ for different values of $\overline{\mathrm{Ric}}$.

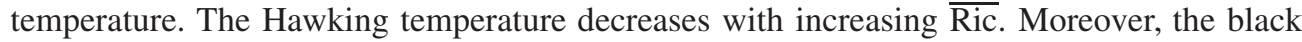
hole remnant in higher $\overline{\text { Ric }}$ has a higher mass. The variation of temperature with $\overline{\text { Ric }}$ shown in figure 5 (right panel).

The thermodynamic details of the quantum gravity system in the scenario of noncommutative geometry investigated respect to heat capacity. It can be obtained by the relation 


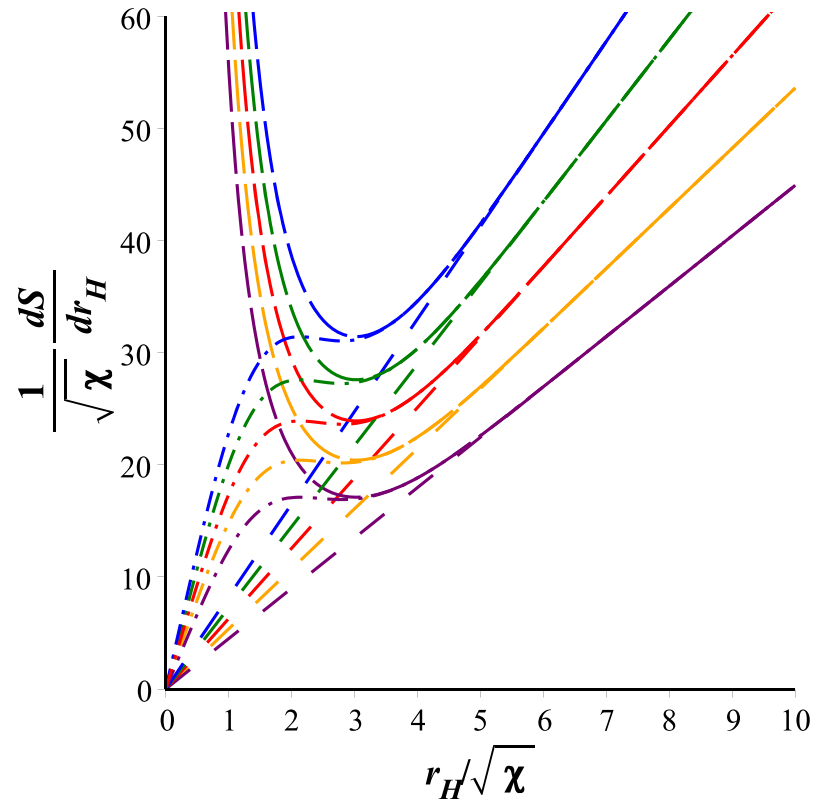

Figure 7. Variation of $\frac{1}{\sqrt{\chi}} \frac{\mathrm{d} S}{\mathrm{~d} r}$ vs $r_{\mathrm{H}} / \sqrt{\chi}$ for the different values of $\overline{\text { Ric }}$. Here, longdash, dashdot and dash linestyle represent noncommutative spacetime, second-order correction of $\frac{\mathrm{d} S}{\mathrm{~d} r}$ and semiclassical limit, respectively.

$$
C=\frac{\partial M}{\partial r_{\mathrm{H}}}\left(\frac{\partial T_{\mathrm{H}}}{\partial r_{\mathrm{H}}}\right)^{-1} .
$$

The behavior of the heat capacity is shown in figure 6 . The heat capacity is positive for $r_{\mathrm{O}}<r_{\mathrm{H}}<r^{\prime}$, which defines small and large black holes are stable. The black holes became unstable for $r^{\prime}<r_{\mathrm{H}}$ due to negative heat capacity. As $r_{\mathrm{H}}$ goes to $r_{\mathrm{o}}$, the heat capacity approaches to zero. For lower $\overline{\mathrm{Ric}}$, the heat capacity is tending to positive, which also defines small black holes are more stable.

From the first law of thermodynamics, the entropy of a black hole can define as $T \mathrm{~d} S=\mathrm{d} M$. Hence,

$$
\frac{\mathrm{d} S_{\mathrm{H}}}{\mathrm{d} r_{H}}=\frac{\pi^{3 / 2} \overline{\mathrm{Ric}}^{3 / 2} r_{\mathrm{H}}}{\gamma\left(\frac{3}{2}, \frac{r^{2}}{4 \chi}\right)} .
$$

The variation is shown in figure 7. The equation (33) can expanded with the help of equation (A.4).

The stability can also be examined by considering the free energy. Free energy can defined as

$$
F=M-T_{\mathrm{H}} S
$$

The numerical calculation of the quantity is shown in figure 8. Positive behavior of free energy supports the stability of a black hole. It is clear from the graph that the free energy 


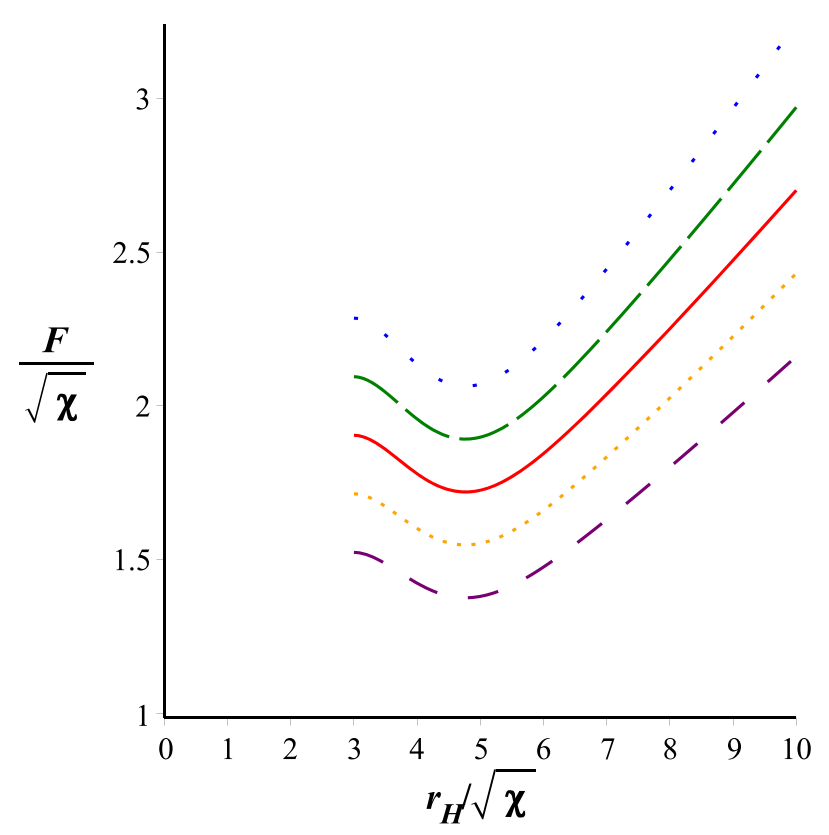

Figure 8. Variation of $F / \sqrt{\chi}$ vs $r_{\mathrm{H}} / \sqrt{\chi}$ for different values of $\overline{\text { Ric. }}$

becomes equivalent to minimum mass at $r=r_{\mathrm{o}}$, thereafter decreases up to $r_{+}$(corresponds to the maximum value of $T_{\mathrm{H}}$ ) and then starts to increases.

\section{Conclusion}

In this article, we have investigated the character and behavior of the noncommutative black hole in the framework of Finslerian spacetime.

In our analysis, we have obtained the relation of the metric potential, temperature, entropy, heat capacity and free energy in trems of Finslerian parameter $\overline{R i c}$. Most interestingly, we observed for the particular Gaussian form of density, the minimal horizon $\left(r_{\mathrm{o}}\right)$ is always at $\simeq 3 \sqrt{\chi}$, for the different minimal masses $\left(M_{\mathrm{o}}\right)$ corresponding to different $\overline{\mathrm{Ric}}$, i.e. the minimal horizon is irrespective of the choice of Finslerian parameter. Due to the negative pressure of the smeared object, it can look similar to the cosmological constant in the de Sitter Universe. In fact, the line element near the origin supports the de Sitter-like behavior inside of the inner horizon $r_{\mathrm{c}}$. It is found that $M_{\mathrm{o}}$ varies with $\overline{\mathrm{Ric}}$, in order of $0.191 \sqrt{\chi}$, also confirmed from figure 4 . Therefore, higher mass accretion can easily explain in the Finslerian background. Hawking temperature increases with decreasing the $\overline{\text { Ric }}$, thought the remnants masses are higher. As the horizon radius reaches the minimal horizon, the temperature of the noncommutative black hole became vanishes. The temperature $\left(T_{\mathrm{H}}\right)$ varies with $\overline{\mathrm{Ric}}$ in a nonlinear manner figure 5 (right panel). Therefore, we can expect that the micro-black holes with large fundamental energy-scale for higher $\overline{\text { Ric }}$ to be hotter, and at the endpoint of evaporation have a smaller mass. From the variation of $\frac{\mathrm{d} S}{\mathrm{~d} r_{\mathrm{H}}}$ vs $r_{\mathrm{H}} / \sqrt{\chi}$, it is found that for second order correction the variation met at $\approx 3 \sqrt{\chi}$, whereas the semiclassical approach met at $\approx 4.8 \sqrt{\chi}$, for the entire range of the Finsler parameter. The decrement of free energy from the minimum mass $\left(M_{\mathrm{o}}\right)$ means the evaporation decelerate in the vicinity of the zero temperature configuration. 
One would consider that the weak energy conditions, $\rho_{\chi}+p_{\mathrm{r}}>0$ and $\rho_{\chi}+p_{\mathrm{t}}>0$ are always satisfied. It is interesting to note that the strong energy condition $\rho_{\chi}+p_{\mathrm{r}}+2 p_{\mathrm{t}}>0$ is not always satisfied. For the region $r<2 \sqrt{\chi}$ strong energy does not obey. This implies, due to the dominance of quantum effects in the region where we supposed, in spite of the nonlinear gravity, the classical description of energy and matter breaks down.

Due to the high temperature at the final stage of black hole evaporation, the effects of backreaction cannot be neglected in commutative background [56-58]. In the noncommutative scenario, the temperature of the final stage is cool enough. Therefore, we can assume that the effects of backreaction should be suppressed. It is observed that the higher $\overline{\text { Ric }}$ reduces the temperature, as a consequence backreaction effects suppressed more intensively, less amount of energy released.

For simplicity, we restricted the system with flag curvature. Following Li and Chang [51], $\overline{\mathrm{Ric}}$ is considered as constant quantity. Akbar-Zahed [50] defines the usefulness of considering constant flag curvature. Such consideration provides us a wide range of spectrum by varying the Finsler parameter $\overline{\text { Ric }}$.

Finally, we can enunciate that the Finslerian parameter provides a range over which the noncommutative black hole can analysis and valid, and the higher order mass accretion the system can easily be explained with the Finslerian background.

\section{Data availability statement}

No new data were created or analysed in this study.

\section{Acknowledgments}

SR and FR are thankful to the Inter-University Center for Astronomy and Astrophysics (IUCAA), Pune, India for providing Visiting Associateship under which a part of this work was carried out. FR is also grateful to Jadavpur University for financial support under RUSA 2.0 and to DST-SERB (EMR/2016/000193), Government of India. SRC and DD are thankful to Debabrata Ghorai and especially SRC is thankful to Ranjini Mondol for the fruitful discussion related to the present work.

\section{Appendix A. Gamma function}

Gamma function can be defined as:

$$
\Gamma(a)=\gamma(s, x)+\Gamma(s, x)
$$

where $\gamma(\mathrm{s}, \mathrm{x})$ know as lower incomplete gamma function and $\Gamma(\mathrm{s}, \mathrm{x})$ as upper incomplete gamma function.

The lower incomplete gamma function is given by

$$
\gamma(s, x)=\int_{0}^{x} t^{s-1} \mathrm{e}^{-t} \mathrm{~d} t
$$

The upper incomplete gamma function is given by

$$
\Gamma(s, x)=\int_{x}^{\infty} t^{s-1} \mathrm{e}^{-t} \mathrm{~d} t .
$$


For $x \gg 1$, the lower incomplete gamma function can expanded asymptotically as follows

$$
\begin{aligned}
\gamma(s, x) & =\Gamma(a)-\Gamma(s, x) \\
& \simeq \frac{\sqrt{\pi}}{2}\left[1-\mathrm{e}^{-x} \sum_{n=0}^{+\infty} \frac{x^{(1-2 n) / 2}}{\Gamma\left(\frac{3}{2}-n\right)}\right] .
\end{aligned}
$$

\section{ORCID iDs}

Sourav Roy Chowdhury (D) https://orcid.org/0000-0003-2802-4138

Farook Rahaman (D) https://orcid.org/0000-0003-4923-7079

Saibal Ray (D) https://orcid.org/0000-0002-5909-0544

\section{References}

[1] Hawking S W 1974 Nature 24830

[2] Hawking S W 1975 Commun. Math. Phys. 43199

[3] Bekenstein J D 1973 Phys. Rev. D 72333

[4] Bekenstein J D 1974 Phys. Rev. D 93292

[5] Aharonov Y, Casher A and Nussinov S 1987 Phys. Lett. B 19151

[6] Susskind L and Thorlacius L 1992 Nucl. Phys. B 382123

[7] Susskind L 1993 Phys. Rev. Lett. 712367

[8] Witten E 1996 Nucl. Phys. B 460335

[9] Seiberg N and Witten E 1999 J. High Energy Phys. JHEP09(1999)032

[10] Snyder H S 1947 Phys. Rev. 7138

[11] Gruppuso A 2005 J. Phys. A: Math. Gen. 382039

[12] Nicolini P 2005 J. Phys. A: Math. Gen. 38 L631

[13] Smailagic A and Spallucci E 2003 Feynman path integral on the non-commutative plane J. Phys. A: Math. Gen. 36 L467

[14] Smailagic A and Spallucci P 2003 UV divergence-free QFT on noncommutative plane. J. Phys. A: Math. Gen. 3611545

[15] Cho S, Hinterding R, Madore J and Steinacker H 2000 Int. J. Mod. Phys. D 09161

[16] Smailagic A and Spallucci E 2004 J. Phys. A: Math. Gen. 377169

[17] Mocioiu I, Pospelov M and Roiban R 2000 Phys. Lett. B 489390

[18] Chaichian M, Sheikh-Jabbari M M and Tureanu A 2001 Phys. Rev. Lett. 862716

[19] Carroll S M, Harvey J A, Kosteleck V A, Lane C D and Okamoto T 2001 Phys. Rev. Lett. 87141601

[20] Ho P M and Kao H C 2002 Phys. Rev. Lett. 88151602

[21] Adorno T C, Gitman D M, Shabad A E and Vassilavich D V 2011 Phys. Rev. D 8484085031

[22] Stern A 2008 Phys. Rev. Lett. 100061601

[23] Vacaru S I 2010 Class. Quantum Grav. 27105003

[24] Connes A 1996 Commun. Math. Phys. 182155

[25] López E 1994 J. Phys. A: Math. Gen. 27845

[26] Madore J 1995 An introduction to Noncommutative Differential Geometry and its Physical Applications (Cambridge: Cambridge University Press)

[27] Heller M and Sasin W 1996 J. Math. Phys. 372665

[28] Heller M et al 1992 Acta Cosmol. 1831 https://ui.adsabs.harvard.edu/abs/1992AcC....18...57H

[29] Heller G, Lambert D and Madore J 1998 Arthroskopie 1151

[30] Castorina P and Zappalá D 2005 AIP Conf. Proc. 756460

[31] Schreck M 2014 J. Phys.: Conf. Ser. 563012026

[32] Nicolini P, Smailagic A and Spallucci E 2006 Phys. Lett. B 632547

[33] Smailagic A and Spallucci E 2002 J. Phys. A: Math. Gen. 35 L363

[34] Myung Y S, Kim Y-W and Park Y-J 2007 Phys. Lett. B 656221 
[35] Banerjee R, Majhi B R and Samanta S 2012 Phys. Rev. D 77 124035x

[36] Banerjee R, Majhi B R and Modak S K 2009 Class. Quantum Grav. 26085010

[37] Rahaman F, Kuhfittig P K F, Chakraborty K, Usmani A A and Ray S 2012 Gen. Relativ. Gravit. 44 905

[38] Radinschi I, Rahaman F and Mondal U F 2013 Int. J. Theor. Phys. 5296

[39] Bao D, Shern S S and Chen Z 2000 An Introduction to Riemann-Finsler Geometry (Graduate Texts in Mathematics) (New York: Springer)

[40] Pfeifer C and Wohlfarth M 2014 Proceedings in Physics (Relativity and Gravitation) ed J Bičák and T Ledvinka (Berlin: Springer) p 157

[41] Kouretsis A P, Stathakopoulos M and Stavrinos P C 2012 Proceedings of M3ST2012

[42] Girelli F, Liberati S and Sindoni L 2007 Phys. Rev. D 75064015

[43] Gibbons G W, Gomis J and Pope C N 2007 Phys. Rev. D 76081701

[44] Hohmann M and Pfeifer C 2017 Phys. Rev. D 95104021

[45] Caponio E, Javaloyes M Á and Masiello A 2011 Math. Ann. 351365

[46] Caponio E, Javaloyes M A and Sánchez M 2011 Rev. Mat. Iberoamericana 27919

[47] Caponio E, Javaloyes M A and Masiello A 2010 J. Phys. A: Math. Theor. 43135207

[48] Vacaru S I 2005 J. Math. Phys. 46042503

[49] Vacaru S, Stavrinos P, Gaburov E and Gonta D 2006 Clifford and Riemann- Finsler Structures in Geometric Mechanics and Gravity, Differential Geometry - Dynamical Systems (Monograph) vol 7 (Geometry Balkan Press)

[50] Akbar-Zadeh H 1988 barb 74281

[51] Li X and Chang Z 2014 Phys. Rev. D 90064049

[52] Chowdhury S R, Deb D, Rahaman F, Ray S and Guha B K 2020 Int. J. Mod. Phys. D 292050001

[53] Lopez-Dominguez J C, Obregon O, Sabido M and Ramirez C 2006 Phys. Rev. D 74084024

[54] Mukherjee P and Saha A 2008 Phys. Rev. D 77064014

[55] Chaichian M, Tureanu A and Zet G 2008 Phys. Lett. B 660573

[56] Balbinot R and Barletta A 1989 Class. Quantum Grav. 6195

[57] Balbinot R, Fabbri A, Frolov V, Nicolini P, Sutton P J and Zelnikov A 2001 Phys. Rev. D 63084029

[58] Balbinot R, Fabbri A, Nicolini P and Sutton P J 2002 Phys. Rev. D 66024014 